\title{
Comparison of Rate of Port Site Wound Infection in Patients Undergoing Arthroscopy Port Closure by Stitch Versus Steri-Strips
}

\author{
${ }^{1}$ Zeeshan Ali Khan, ${ }^{2}$ Benash Altaf, ${ }^{2}$ Shireen Jawed, ${ }^{3}$ Sadaf Altaf \\ ${ }^{1}$ Department of Orthopedics, DHQ Hospital, Faisalabad \\ ${ }^{2}$ Department of Physiology, Aziz Fatimah Medical and Dental College, Faisalabad \\ ${ }^{3}$ Department of Gynecology \& Obs., Social Security Hospital, Faisalabad
}

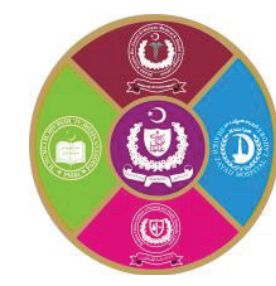

\section{ABSTRACT}

Introduction: Arthroscopy is a surgical procedure which is used to visualize, diagnose, and treat various problems of joints. This minimal invasive procedure is gaining its importance because of using a small incision for introducing camera in the knee joint. However proper suturing of that incision is also needed for good outcomes of arthroscopic surgery. Although various methods of suturing have so far been discovered but it is still debatable which suturing method is to be opted by surgeons to avoid complications. Aims \& Objectives: To compare the infection rate among the patient's arthroscopic portal wound closure by steristrip and suture technique. Place and duration of study: This was quasi experimental study conducted at orthopedic department of private Faisalabad based hospital, from $1^{\text {st }}$ May to $31^{\text {st }}$ July 2018. Material \& Methods: The study included 128 patients who underwent arthroscopy of knee joint, either for diagnostic purposes or for therapeutic procedures. The procedure was performed by following standard protocols and portal site wound was either closed by steri-strip method or by suture technique. All patients were assessed for post-operative wound infection on $10^{\text {th }}$ post-op day. Infection rate was compared among the patients with wound closure by setri-strip and sutured technique (prolene 2/0) admitted as a day cases. Outcome measure adopted was grade of infection at port site. Data was analyzed by SPSS 21.0. Infection rates are presented as percentages and compared by Chi-square test $\left(\mathrm{X}^{2}\right)$. Results: Study revealed that in 128 patients $(89.1 \%$ male and $10.9 \%$ female), overall infection rate followed by arthroscopy was $6.3 \%$. These results also revealed equal infection rate for both procedures. However severity of infection was seen more severe in stitch opted method in contrast to steri-strip. Conclusion: Both methods for wound closure are equally good and have no difference for rate of infection at port site. The present study shows that suturing the portals has no additional benefit and treating these wounds with steri-strip is easy, cost effective and is comfortable to subject.

Key words: Wound, stitch, steri-strip, arthroscopy

\section{INTRODUCTION}

K nee arthroscopy is the frequently performed orthopedic technique which is being done for the diagnosis and management of knee disorders this technique help to inspect and manipulate the whole meniscus and recognize the extent of the lesion prior to excision. This technique is done mostly by making anterolateral and anteromedial ports. With the use of specialized instruments in this technique make possible to excise lesion in any part of the meniscus. ${ }^{1}$

In order to minimize and avoid the portal site wound infection, procedures under proper aseptic measures should be opted to close the wound sites. ${ }^{1}$ It is believed that thin soft tissue like a membrane envelopes the knee joint which is responsible to increase incidence of wound healing related complications, hence, there are different newly evolved methods for closure of these portalsin daily practice by orthopedic surgeons. ${ }^{2}$ These include from absorbable sutures ,non-absorbable nylon sutures, bow tie stitch, surgical tapes, adhesive gels, sterile strips, or blends of above mentioned techniques., ${ }^{2,3}$ Even some surgeons reported equal fruitful results when they left the port site wound opened. ${ }^{4}$ Though, research evidences are available showing the superiority of one method over the other ${ }^{5,6}$ but, choice for closing the portal site greatly depends on the surgeon's inclination towards individual subjective care. Contradictory studies have been documented in previous literature concerning effective methods of wound closure which are believed to be helpful in quick recovery 
and also helpful in prevention of further complications.

Aim of current study is to compare commonly practiced methods of skin closure in patients having knee arthroscopy with a single prolene suture and closure with the use of steri-strips in term of infections rates. We also aimed to determine the frequency of patients with various knee problems coming for arthroscopy.

\section{MATERIAL AND METHODS}

This Quasi experimental study was performed at private hospital in Faisalabad from $1^{\text {st }}$ May to $31^{\text {st }}$ July, 2018. 128 patients admitted in this duration for arthroscopy were enrolled in this study. Relevant information about patients was recorded on predesigned Proforma. Inclusion criteria were patients coming for reconstruction of anterior cruciate ligament, minisectomy, synovial biopsy for diagnostic purpose. Patients with septic arthritis were excluded from the study. Prior to procedure informed consent was taken. Patients were divided in two groups on the basis of wound closure techniques (prolene 2/0 single stitch $\mathrm{v} / \mathrm{s}$ steri-strips). All patients underwent arthroscopy for diagnostic or therapeutic purposes through 2 port techniques, using a tourniquet $(400 \mathrm{mmHg}$ pressure for 45 minutes). Ports of one group were closed by prolene 2/0 single stitch while other group with steri-strips. Mean duration of the procedure was about 45 minutes. All procedures were done under spinal anesthesia in supine position and controlled tourniquet application for safe time. No preoperative antibiotics were given. At the end of procedure, local anesthetic was applied topically after wound closure. However, post-operative antibiotics 3 oral doses at 8 hours interval were given to all patients. Each patient was assessed for infection like, cellulitis and lymphadenitis on 10th postoperative follow up day on the basis of presence of signs like redness, tenderness, and edema for these infections.

\section{Statistical analysis:}

Data was analyzed using SPSS 20. Descriptive of the study population are presented as mean and standard deviation (SD). Infection rates are expressed as frequencies and percentages. Chisquare was applied to compare the proportions. $\mathrm{P}<0.05$ was considered statistically significant.

\section{RESULTS}

128 patients were enrolled in this study with mean age $31.0 \mathrm{SD} \pm 10.94$. Out of this, $114(89.1 \%)$ were male subjects and $14(10.9 \%)$ were female (Figure1). Enrolled subjects for arthroscopy coming with knee problem was presented most commonly with meniscal injury $(79.5 \%)$, followed with anterior cruciate ligamental injury $(11 \%)$ and then arthritis $(7.9 \%)$ least common presentation was impingement syndrome(1.6\%). Figure-2

$64(50 \%)$ subjects have wound closure with steristrip and the same percentages of the subjects had wound closure with stitch method. Overall infection rate was $6.3 \%$ i.e total 8 subjects found to have infection including cellulitis and lymphadenitis. Table-1 is indicating that that overall infection rate between the steri-strip closure method and stitch closure method (studied groups) is same (6.3\%). Of total subjects in steris-strip method $4.6 \%$ of subjects develop cellulitis and only $1.6 \%$ develop lymphadenitis in contrast to subjects closed with stich method who develop cellulitis and lymphadenitis equally. These results also reveal that infections caused by steri-strips were less severe than stitch closure methods. (P value 0.693) $\mathrm{P}$ value $\geq .05$ is considered as significant.

\begin{tabular}{|c|c|c|c|c|}
\hline $\begin{array}{l}\text { Total } \\
\text { case }\end{array}$ & \multicolumn{3}{|c|}{ Steri strip closure method } & $\begin{array}{c}P \\
\text { value }\end{array}$ \\
\hline \multirow{7}{*}{64} & \multicolumn{2}{|c|}{$\begin{array}{c}\text { Infection cases } \\
\mathrm{N}(\%) \\
\end{array}$} & \multirow{2}{*}{$\begin{array}{c}\text { Non } \\
\text { infective } \\
\text { cases } \\
\mathrm{N}(\%) \\
\end{array}$} & \multirow{7}{*}{0.693} \\
\hline & cellulitis & lymphadenitis & & \\
\hline & $3(4.6 \%)$ & $1(1.6 \%)$ & $\begin{array}{c}60 \\
(93.8 \%) \\
\end{array}$ & \\
\hline & \multicolumn{3}{|c|}{ Stitch closure method } & \\
\hline & \multicolumn{2}{|c|}{$\begin{array}{c}\text { Infection cases } \\
\mathrm{N}(\%) \\
\end{array}$} & \multirow{2}{*}{$\begin{array}{c}\text { Non } \\
\text { infective } \\
\text { cases } \\
\mathrm{N}(\%)\end{array}$} & \\
\hline & cellulitis & lymphadenitis & & \\
\hline & $2(3.15 \%)$ & $2(3.15 \%)$ & $\begin{array}{c}60 \\
(93.8 \%) \\
\end{array}$ & \\
\hline
\end{tabular}

Table-1: Comparison of infection rate by steri-strip and stitch closure method

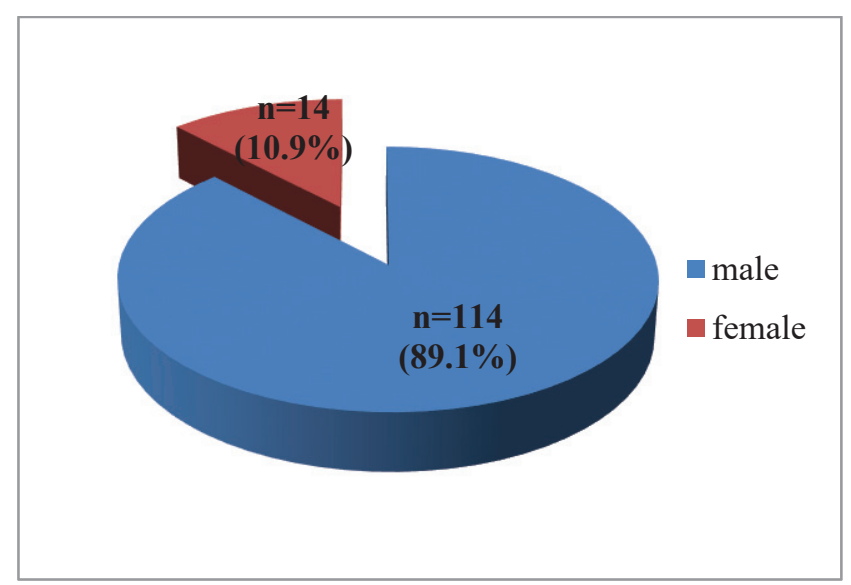

Fig-1: Distribution of gender among the studied group 


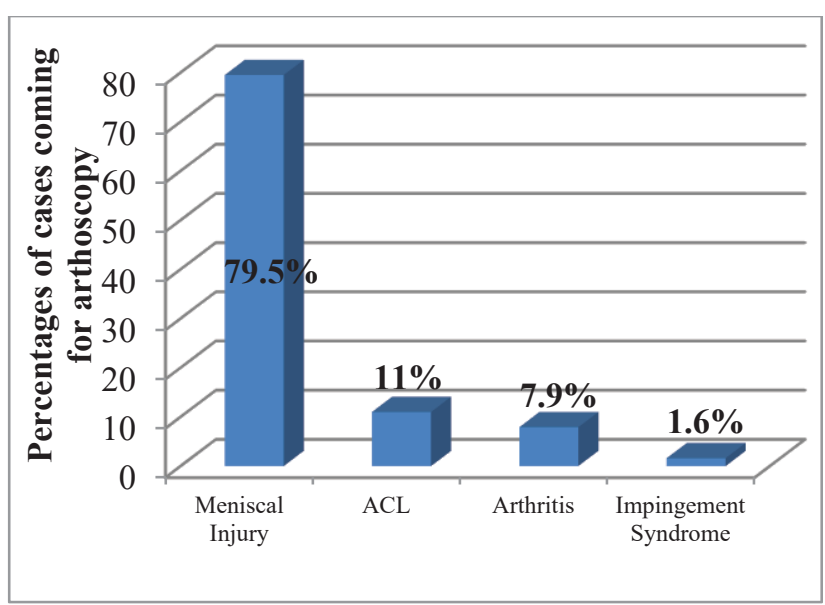

Fig-2: Subjects for arthroscopy with various knee problems

\section{DISCUSSION}

Knee osteoarthritis and ligamental injuries are rising by increasing average age of general population resulting in disability. ${ }^{7}$ Knee arthroscopy is common orthopedic surgical procedure frequently performed for diagnosis and treatment of wide range of knee disorders. It is widely considered to be a safe procedure but still it is associated with portal site wound infections, deep vein thrombosis, pulmonary embolism, and even deaths. ${ }^{7}$ It is imperative to expand updated information on safety of the procedure. Techniques followed for wound closure after arthroscopy has great importance to prevent the portal site wound infections. In our study most common presentation for knee arthroscopy was meniscal injury (79.5\%), followed by anterior cruciate ligamental injury (11\%) and then arthritis (7.9\%) least common presentation was impingement syndrome (1.6\%). Portal site wound infections are still common and the wound closure method is most common contributing factor in causing these infections. Very few documented studies are available for comparing the risks of complications followed by wound closure techniques. This study was conducted to highlight the different methods proposed for portal site wound closure. Proper skin approximation and reduce tension on portal spot site is required for better healing purposes and outcomes. To attain these outcomes, various procedures have been proposed like wound drainage, application of tension on edges of the wound, enveloping of soft tissues, nylon and absorbable thread suturing, and closure with steristrips and adhesive tapes. ${ }^{8}$

Various researches are available showing that reduction in wound complications in knee arthroscopy is attributed to portal site suturing ${ }^{8}$ Fair clough and his colleagues had reported loftier results by using adhesive tape and steri strips versus sutures in knee arthroscopy portals ${ }^{9}$. Many other researchers also found similar results with decrease rate of wound infection and inflammation with taped closure. However Maffulli etal. study documented less wound complications with use of sutureless technique for wound closure ${ }^{11}$. Regading the wound closure techniques, previous researchers also documented conflicting results. Current study did not find any difference concerning the infection rates following steri-strip and the sutured technique for closure of arthroscopic knee wound porches. However, infection found in steri-strip method was less in severity than sutured technique. $4.6 \%$ and $1.6 \%$ of subjects develop cellulitis and lymphadenitis respectively followed by steri-strip closure in contrast to this $3.15 \%$ subjects closed with sutured techniques got lymphadenitis. This indicates that infections caused by sutured techniques are more common and severe.

These results are in accordance to studies conducted by Zempsky etal and, Tritle etal in past who also showed no differences in wound healing done with the adhesive tape, absorbable suture and nylon sutures regarding portal site wound infection outcome $^{12,13}$. Our study also valued both techniques equally, supporting previous studies but this is also worth noticeable that when we compared Infection rate in steri-strip vs stich closure method then we found equal percentages $(6.3 \%)$ for each method.

However, adhesive tape technique, used in this study showed the superiority in regard that it is cost effective. But, further experimental procedures and time is required to depict the growing importance of this technique. However, Bunker was the one who focused and pointed out problems, that was managed and avoidable by using sutureless tecniques. ${ }^{14}$

Current study showed over all infection rate of $6.3 \%$ following closure of wound by stitch and steristrips techniques which is in close appoximty to the results documented by Pajalic KFas $5 \% .{ }^{15}$ No significant difference for infection rate was observed between these two closing wound methods. However $\leq 1 \%$ wound infection rate especially for septic arthritis following knee arthoscopies have been reported by previous studies. ${ }^{15}$ Despite of these decrease infection rates, in unfortunate cases of a deep infection, a prolonged postoperative IV antibiotic treatment is required. So, in current study, keeping that infection rate in mind, cortisone was castoff in all patients as a protective measure to minimize and reduce the inflammation and infection due to its anti-inflammatory properties. Despite of using this prophylactically in 
our study infection rate is documented $3.1 \%$ cellulitis and 3.1\% lymphadenitis other than over all low rate of infection in the studied population. Studies are available showing that cause of this infection may be due to application of tourniquet for unsafe time period. Previous literature showed that this may be due to the resulting ischemia leading to infection. ${ }^{1,16}$ This finding is inconsistent with our study. We found less infection rate using tourniquet, applied for controlled time (45 minutes) which is considered as a safe time period. Only four patients in each group were affected among the all patients who underwent surgery under time controlled application of tourniquet, probably showing that ischemia is not the sole cause for infection in our study. ${ }^{17}$ No previous data was found showings calated infection rate with use of tourniquet within safe time period. So, this was novel in our study that we used a joint parameter using, both the safe duration of tourniquet use and the pressure applied. Strength of the current study is that we tried to reduce prejudice attitude towards a specific technique by surgeons participating in the study. However, there were chances for bias towards reporting superior results for their personal/private cases. So, this bias was controlled by adopting inclusion criteria including additional hospital admissions, a validating scoring system and use of antibiotics. Weakness of the study is that for each procedure whether diagnostic or therapeutic, the postoperative immobilization and weight bearing status was not the same. Additionally recording of healing outcomes was variable with dearth of independent observation in grading of wound.

\section{CONCLUSION}

Both procedures are found effective for healing the wound and show good cosmetic results. However choosing sutureless technique preferably, avoids physical trauma in contrast to wound closed with stitch. Moreover it is cost effective.

\section{REFERENCES}

1. Ward BD, Lubowitz JH. Basic knee arthroscopy part 2: surface anatomy and portal placement. Arthrosc Tech. 2013; 22:2(4):501-2.

2. MS Sajid, MR McFall, etal. World J Gastrointest Surg. 2014; 6(12): 241-247.

3. S Peter, Panagiotis D. Symeonidis, Lukas D etal. Sutures Versus Sterile Strips for Closure of Ankle Arthroscopy Portals: Prospective Crossover Trial. Foot \& Ankle International. March 2012; 33: 190.
4. M R Chetan, K Murali etal, No differences in healing among different closure methods of arthroscopic portals: a systematic review, 2017.

5. Sikand, M; Murtaza, A; Desai, VV: Healing of arthroscopic portals:a randomised trial comparing three methods of portal closure. Acta Orthop Belg, 2006; 72:583.

6. Nacius LA, Pascual-Garrido C, Sagiv P, MeiDan O. Skin closure after arthroscopy utilizing a pull-out bow-tie subcuticular suture. Muscles Ligaments Tendons J. 2015; 5; 4(4):404-6 7.

7. Heidari B. Knee osteoarthritis prevalence, risk factors, pathogenesis and features: Part I. Caspian J Intern Med. 2011 Spring;2(2):205-12.

8. K P Paras, S Ashish, K Ashwani, etal . A Comparative Study of Wound Closure with Disposable Skin Stapler Versus Conventional Sutures. Int J Med Res Prof. 2017; 3(2); 102-06.

9. Fairclough, JA; Moran, CG: The use of sterile adhesive tape in the closure of arthroscopic puncture wounds: a comparison with a single layer nylon closure. Ann R Coll Surg Engl 1987; 69:140

10. Conolly, WB; Hunt, TK; Zederfeldt, B; et al.: Clinical comparison of surgical wounds closed by suture and adhesive tapes. Am J Surg. 1969; 117:318.

11. Maffulli, N; Pintore, E; Petricciuolo, F: Arthroscopy wounds: to suture or not to suture. Acta Orthop Belg 57:154, 1991

12. Zempsky, WT; Zehrer, CL; Lyle, CT; et al.: Economic comparison of methods of wound closure: wound closure strips vs. sutures and wound adhesives. Int Wound J, 2005; 2:272.

13. Tritle, NM; Haller, JR; Gray, SD: Aesthetic comparison of wound closure techniques in a porcine model. Laryngoscope, 2001; 111:1949.

14. Bunker, TD: Problems with the use of Op-Site sutureless skin closures in orthopaedic procedures. Ann R Coll Surg Engl, 1983;65:260

15. Pajalic KF, Turkiewicz A, Englund $M$ et al. Update on the risks of complications after knee arthroscopy BMC Musculoskeletal Disorders (2018) 19:179

16. T.Bauer, P.Boisrenoult, J.-Y.Jenny, Postarthroscopy septic arthritis: Current data and practical recommendations. Orthopaedics \& Traumatology: Surgery \& Research Volume 101, Issue 8, Supplement, December 2015, Pages S347-S350.

17. P Rocco, Z Biagio. Tourniquet in knee surgery. British Medical Bulletin, 2014; 111: (1),1,63-76. 


\section{The Authors:}

Dr. Zeeshan Ali Khan,

Dr. Sadaf Altaf,

Senior Registrar,

Orthopedic Department,

Women Medical officer,

DHQ Hospital, Faisalabad.

Department of Gynecology \& Obs.

Social Security Hospital, Faisalabad

Dr. Benash Altaf,

Assistant Professor,

Department of Physiology,

Aziz Fatimah Medical and Dental College, Faisalabad.

\section{Corresponding Author:}

Dr. Zeeshan Ali Khan,

Senior Registrar,

Orthopedic Department,

DHQ Hospital, Faisalabad.

Dr. Shireen Jawed,

E-mail: zeeshanalikhan67@gmail.com

Associate Professor,

Physiology Department,

Aziz Fatimah Medical College, Faisalabad. 